\title{
LA RELACIÓN INTRAPERSONAL: PRIMER PILAR DE LA RELACIÓN DE CUIDADO. EXPERIENCIAS Y REFLEXIONES DEL CAMPAMENTO DE SALUD MENTAL
}

Andrés David Bernal Galvis¹, Vanessa Margarita León Ospina¹N Nicol Vanessa Posada Sánchez¹, Andrés Steven Ibagué Peralta1 ${ }^{1}$, Jack Bejarano Duarte ${ }^{1}$, Sandra Vibiana Carvajal Muñoz ${ }^{1}$

\section{Resumen:}

La relación intrapersonal, entendida como la capacidad de observar las emociones, ideas y comportamientos en un contexto particular y de manera histórica (Cooper, 2003), implica sistemáticos procesos de autorreflexión en un diálogo consigo mismo (Alonso, 2000) que produce un estado de ánimo o tono emocional, así como el desarrollo de la confianza y serenidad propia en la vida cotidiana y los compromisos que se puedan adquirir (Rogers y Wainberg, 2014). En la construcción de esta relación, se manifiesta un cuidado de sí mismo que resuena en la pregunta orientadora del primer semestre del pregrado de Enfermería de la Fundación Universitaria Juan N. Corpas y que se busca enfatizar a lo largo de la carrera. En el marco del hilo conductor de Cuidado y Calidad de Vida, la salud mental se reconoce, en primer lugar, como un estado vital del estudiante, docente y, en general, de la comunidad académica. En consecuencia, al plantear el desarrollo de los contenidos académicos requeridos para la carrera de Enfermería, se ubica la salud mental del estudiante como centro, lo que estimula a comprometer algunas estrategias de la formación que impliquen el desarrollo humano.

En este marco, durante el semestre 2021-2022, se realizó el Campamento de Salud Mental con estudiantes de primer semestre, en el que se enfatizó en la relación intrapersonal. El presente artículo presenta una de las experiencias centrales de este, Ilamado "Esculturas colectivas", a partir de las reflexiones de los y las estudiantes que participaron en el campamento. Finalmente, se plantean conclusiones construidas en comunidad, con el equipo facilitador del campamento.

Palabras clave: percepción, toma de decisiones, emociones.

Historial del artículo:

Fecha de recibido: 08-09-2021 - Fecha de aceptado: 07-10-2021

(c) 2021 Fundación Universitaria Juan N. Corpas. FUJNC.

Artículo Open Access bajo la Licencia Creative Commons Atribución-NoComercial-Compartirlgual 4.0 Internacional.

DOI: https://doi.org/10.26752/revistaparadigmassh.v3iSupl.1.608

${ }^{1}$ Estudiantes de primer semestre de Enfermería 2021-2022. Fundación Universitaria Juan N. Corpas. Bogotá, D.C., 


\title{
INTERPERSONAL RELATIONSHIP: FIRST PILLAR OF CARE, RELATIONSHIP, EXPERIENCES AND REFLECTIONS OF MENTAL HEALTH CAMP
}

\begin{abstract}
:
The intrapersonal relationship understood as the ability to observe their emotions, ideas and behaviors in a particular context, and historically (Cooper, 2003), implies systematic processes of self-reflection in a dialogue with oneself (Alonso, 2000) that produces a state of mood or emotional tone, and the development of the confidence and serenity of counting on oneself in daily life and for the commitments that may be made (Rogers and Wainberg, 2014). In the construction of this relationship, self-care is visible that is in the guiding question of the first semester of the nursing course and that will seek to emphasize semester by semester within the framework of the guiding thread of Care and Quality of Life, in the which is recognized, first of all, mental health as a state of the student, teacher and the academic community in general. Consequently, when proposing the development of the academic content required for the discipline of Nursing, the mental health of the student is placed as the center, which encourages them to compromise other training strategies that involve human development.

In this framework, during the 2021-2 semester, the Mental Health Camp was held with first-semester students, about the intrapersonal relationship. This article presents one of its central experiences, called: collective sculptures; from the reflections of the students who participated in the camp, raising conclusions co-constructed with the facilitating team of the camp.
\end{abstract}

Keywords: perception, decision making, emotions. 


\section{Introducción}

El Proyecto Educativo de Programa (PEP) de la Fundación Universitaria Juan N. Corpas (Román et al., 2015), en el apartado de competencias generales -que se refiere al desarrollo personal, el cual está alineado con la misión del Proyecto Educativo Institucional (Piñeros Ricardo et al., 2019) y resalta la contribución al desarrollo de la persona de manera integral y pertinente con sentido humanístico, ecológico y social dentro del marco de la excelencia-, ha establecido un hilo conductor de Cuidado y Calidad de Vida, en el cual se reconoce la salud mental como un estado vital del estudiante, docente y en general de la comunidad académica.

Así, en el ciclo de fundamentación, que ocurre durante los primeros tres semestres, se realizan los Campamentos en Salud Mental ${ }^{2}$, en los que se privilegia la vivencia interna como fuente de aprendizaje; en estos, el estudiante conoce y fortalece su estructura psíquica, en el camino de dar sostén mental para sí mismo, y desde allí recibe y organiza nuevos contenidos en los que profundiza, a fin de desarrollar habilidades para atender la salud mental de las personas requirentes de cuidado, en especial, aquellos que implican mayor complejidad. En dichos semestres, se abordan tres relaciones: la intrapersonal, la interpersonal y los lazos sociales.

Como se mencionó anteriormente, la relación intrapersonal es entendida como la capacidad de observar las emociones, ideas y comportamientos (Cooper, 2003), que traen consigo cambios en el desenvolvimiento cotidiano, tanto personal como colectivamente. En la construcción de esta relación, se visibiliza un cuidado de sí mismo que está en la pregunta orientadora del PEP, cuyo hilo conductor procura construir fundamentos en los y las estudiantes que conlleven al cuidado de sí mismo y de los otros. Desde la perspectiva genealógica de Foucault, el cuidado de sí se entiende como un conjunto de prácticas, mediante las cuales un individuo establece cierta relación consigo mismo; así, en esta relación el individuo se constituye en sujeto de sus propias acciones (Giraldo y Zuluaga, 2013).
Según el psicoanálisis, todo proceso psíquico implica la relación consigo mismo y la comprensión de las fuerzas a las que está sometida la mente del ser humano. En el proceso del autoconocimiento, el ser humano identifica la forma de afrontar las exigencias del mundo exterior y de su mundo interior, la relación entre ambos y el sufrimiento psíquico, entre otros aspectos. Uno de los aspectos relevantes en el autoconocimiento es la causa del sufrimiento psíquico, dado que este tiene la capacidad de impulsar el dinamismo interior; Freud (1984) atribuye el sufrimiento a tres fuentes (la naturaleza, la realidad y las relaciones), ubicadas en el mundo exterior. Paradójicamente, la mitigación de dicho sufrimiento no se encuentra al intervenir en el mundo exterior, sino en el mundo interior.

En lo que se conoce como la Prevención del Burnout, Nespeira-Campuzano y Vasquez-Campo (2017) enfatizan en que "las emociones influyen notoriamente en el proceso curativo del paciente, ya que estas se consideran componentes esenciales en la salud de la persona" (p. 176). Estas transacciones emocionales, calidez y comprensión, que involucran al profesional de enfermería, exigen inteligencia emocional para afrontar con mayor solvencia las exigencias que el trabajo plantea, en el que se destaca que la claridad y la reparación emocional tiene una relación de alto nivel de significancia al momento de encontrar la realización laboral.

Por su parte, la relación de cuidado en el ejercicio profesional y el aspecto psicodinámico del trabajo en enfermería - expuesta por Dejours y retomada en particular para el trabajo de cuidado por diversas autoras como Molinier, Wlosko, Ros, entre otras (citadas en Borgeaud-Garciandía (2018))-, develan la gran exigencia psíquica que conlleva la construcción de relaciones de cuidado por los y las profesionales de enfermería. De esta forma, como parte de la formación durante el pregrado, se requiere brindar elementos para la construcción de dichas relaciones; en el primer semestre, en particular, la Escuela de Enfermería de la Fundación Juan N. Corpas orienta dicho trabajo a la consecución de la relación intrapersonal, sin la cual los demás componentes de las

${ }^{2}$ A la fecha, la experiencia del Campamento de Salud Mental dura veinticuatro horas y aborda: (a) la mente en el cuerpo; (b) la teoría de la mente como base de la empatía; (c) toma de decisiones y superación de la dicotomía emoción-razón, y (d) el autoconocimiento como tarea fundamental y permanente. 
relaciones de cuidado no tendrían lugar, pues, si no hay sujeto, ¿cómo encontrarme con el otro/a, sean colegas, miembros del equipo de salud o las personas que se atienden? (Piñeros Ricardo et ál., 2019).

Durante el semestre 2021-2022, se realizó el Campamento de Salud Mental con estudiantes de primer semestre, acerca de la relación intrapersonal. A continuación, se presenta una de las experiencias de dicho encuentro, "Esculturas colectivas", la cual suscitó las reflexiones de los y las estudiantes que participaron.

Esculturas colectivas: Campamento de Salud Mental de la asignatura Cuidado y Calidad de vida (14, 15 y 16 de agosto de 2021)

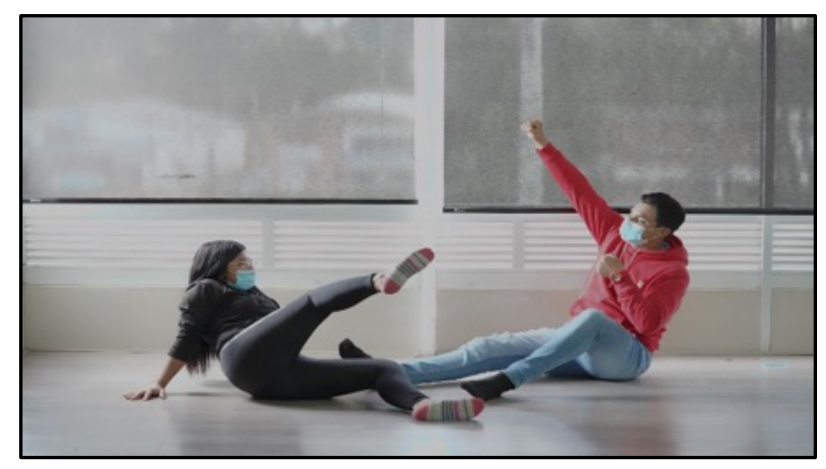

Nombre de la escultura: "La chalaca y el helado" Creadores/as: Kenny Javier Fonseca Trujillo, Linda Lucía López Perdomo. Sandra Vibiana Carvajal Muñoz

\section{Descripción:}

Somos cuerpos diferentes, pero el bienestar es un factor común: si estamos bien emocionalmente, podemos tener la capacidad de actuar mejor ante los demás. Las relaciones intrapersonales nos facilitan las relaciones con otros, al ayudar de forma mutua al crecimiento intrapersonal; así, podrá haber un mejor y más eficaz trabajo para poder sobrellevar las exigencias del exterior.

En la escultura, se aprecian un hombre y una mujer que tienen un vínculo afectivo: la amistad. Se encuentran en un entorno recreativo, relajados, sin afán ni preocupaciones; solo disfrutan del momento. Ella está practicando fútbol y demuestra su pasión por él; esa disciplina, ese sacrificio, esa pasión, se reflejan en su cuerpo, así como su amor, dedicación y alegría, al realizar una maniobra física y golpear un balón con el pie. A la jugada, en este caso, se le llama chalaca. Se observa muy cerca de ella a un él: un hombre, un amigo, un confidente, que comparte con ella un gusto en común, y que demuestra su apoyo a su amiga, su compañera, con un delicioso helado.

\section{Aprendizaje}

1. Todos podemos llegar a expresar nuestras emociones.

2. Todos las expresamos de maneras diferentes.

3. A pesar de los múltiples defectos que tenemos, las cualidades pueden ayudar a compensarlos.

4. Todos podemos llegar a necesitar ayuda de un/a profesional en algún momento de nuestras vidas.

5. Podemos llegar a controlar y mejorar la manera de expresarnos ante el mundo.

6. Cada ser humano tiene una lucha interna, por eso debemos ser siempre solidarios.

7. Somos un manojo de emociones sin explorar.

8. Estamos en continua evolución del reconocimiento del verdadero yo.

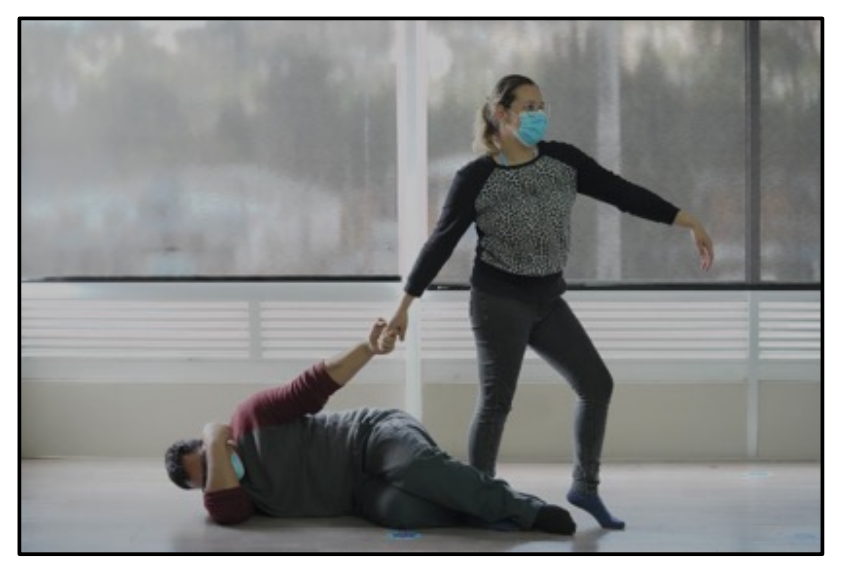

Nombre de la escultura: "Desapego" Creadores/as: Andrés David Bernal Galvis, Nicole Vanessa Escalante, Fabián Camilo Díaz Kelly Stefany Ayala 


\section{Descripción}

La escultura representa la necesidad que tenemos como individuos de identificar lo positivo y negativo que nos brindan las personas de nuestro entorno. Así mismo, la potencia que tenemos como seres racionales de recibir aquello positivo y desechar lo negativo, lo que nos convierte en los responsables del manejo de nuestras emociones, al tener en cuenta que todas nuestras decisiones tienen consecuencias y que nos pueden afectar física, emocional y psicológicamente.

Está ligado directamente con el amor propio, pues sé que, si yo me amo, me respeto y me valoro, puedo permitir o no aferrarme y dejarme afectar por las cosas negativas; Puedo optar por tomar de ellas solo aquello que me permita fortalecer mi camino, que favorezca mi crecimiento como persona y que guíe mis sueños y propósitos.

\section{Aprendizaje}

Nos llevamos una gran experiencia, ya que tuvimos la posibilidad de interactuar como grupo desde un ámbito diferente al académico, lo que nos permitió conocer un poco más de nuestros compañeros. De igual manera, adquirimos herramientas para regular o manejar nuestras emociones, a creer en nosotros mismos y saber quiénes somos en realidad para lograr llegar a nuestra meta individual.

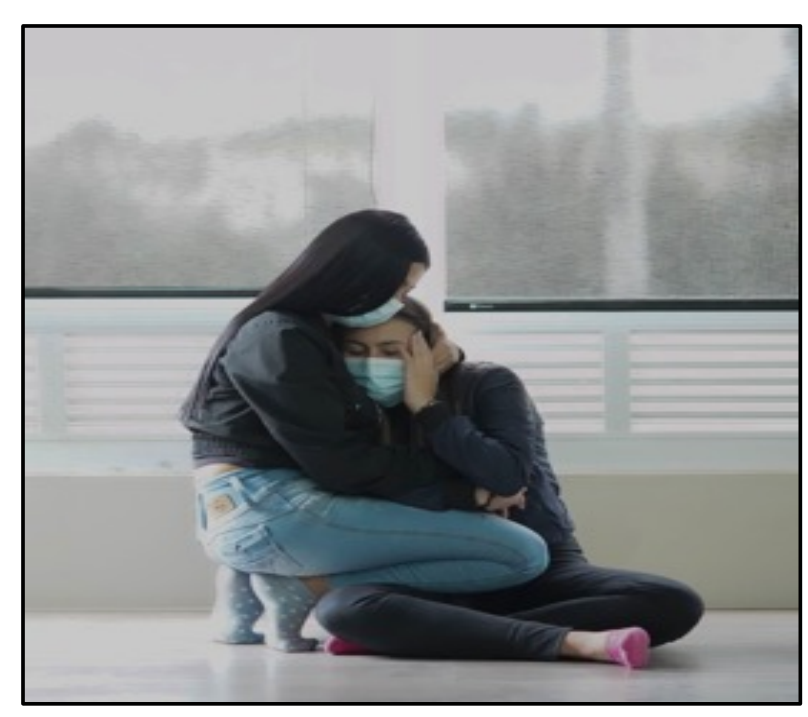

\section{Nombre de la escultura: "Compasión"}

Creadores/as: Jack Bejarano Duarte, Danna Valeria Solano Briñez, Nicol Sofía Quimbayo Rodríguez, Sharon Arlene Quimbayo Rodríguez

\section{Descripción}

En la escultura, se reflejan varios sentimientos juntos, como el consuelo, el amor, la seguridad y la tristeza. Estas son emociones que van conectadas a nuestros pensamientos y nuestras acciones, lo que da como resultado una emoción interactiva con las personas alrededor del entorno. Esto es lo que expresa la escultura: no una emoción, sino varias y su respectivo significado al verla; mirar a profundidad los rasgos físicos que pueda darnos una similitud, de lo que significa aquella. La escultura tiene una historia muy conmovedora: trata de dos hermanas que tienen un vínculo amoroso, la hermana pelinegra está consolando a su hermana que sufre de esquizofrenia - una enfermedad mental que puede causar cambios como diferentes personalidades, emociones y escuchar voces-, algo que ella no puede controlar, pero gracias al apoyo y seguridad que le da la hermana puede afrontar y combatir sus emociones negativas e interactuar con las personas para así tener conexiones afectivas con ellas.

\section{Aprendizaje}

Aprendimos a conectarnos con el pasado, para revivir esos momentos alegres y tristes; algunos compañeros pasaron momentos muy tristes y les sirvió desahogarse y quitar ese pensamiento negativo que vivieron en el pasado. Pudimos evidenciar que la tranquilidad es un reflejo que podemos dar a la sociedad, que no tenemos que agachar la mirada, sino observar el mundo con una perspectiva emocional e interactiva; mirar lo que la gente demuestra con su rostro, dar una mirada crítica de las emociones del entorno que conforma un individuo. En el campamento, aprendimos que la seguridad, la confianza y las emociones van conectadas entre sí, lo que da como resultado un propósito de vida y los sueños que con dedicación se pueden volver realidad. Que hay que intentar y no rendirnos, a pesar de las adversidades que haya, luchar por las cosas que realmente queremos y mostrar respeto a los demás y a nosotros mismos, recordar que una buena postura y un buen equilibrio nos permiten tener más presencia y más seguridad cuando expresamos nuestros sentimientos. 


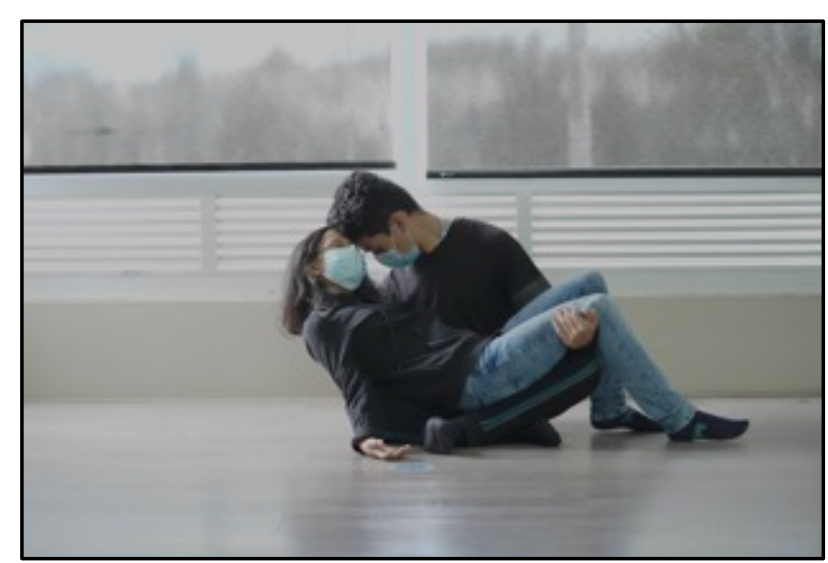

\section{Nombre de la escultura: "El amor a la muerte"}

Creadores/as: María Camila Afanador Triana, Mayerly Santamaría Jeréz, Astrid Alejandra Núñez Gáfaro, Esteban Espitia Cubillos

\section{Descripción}

La forma del sentimiento, basado en el arte, es caracterizada por el dolor de la pérdida, por el amor al tiempo y el odio a la muerte. Se sabe que el ser humano se aferra a las emociones y las transforma en un aspecto relativo a su salud, tanto física como mental; por ello, es necesario que el humano posea paz mental y espiritual. Sin embargo, existen circunstancias que afectan estos procesos de superación e interrumpen en el camino de la armonía; aun así, el dolor, la tristeza y el llanto son esenciales para poder llegar a este sendero, pues nuestro pasado conlleva emociones de todo tipo, como alegría, tristeza, dolor, miedo, euforia. Nuestra escultura evidencia la pérdida: el amor es consumido por el odio y el dolor. Muchos verán estas emociones como negativas, pero, según nuestra manera de pensar, el odio lleva al perdón y el dolor a la paz; la vida no está diseñada para vivirse de una manera monótona. Los sucesos ocurren por un motivo y nosotros representamos la paz mental y su salud, a través del dolor.

\section{Aprendizaje}

El campamento fue diseñado para buscar la paz y armonía que perdemos con el paso de los años y nos enseñó a identificar cada paso, hasta poseer la tranquilidad que nos fue arrebatada por las emociones negativas. En el reconocimiento del pasado, el cual, para algunos, fue un momento muy importante que se ve reflejado en su personalidad, pudimos recordar y lidiar con las emociones que nos aferran a él. Además de esto, concluimos la importancia de centrarnos en nosotros mismos, cuidarnos y, lo más importante, que es prestar atención a nuestra salud mental. La enseñanza fue más de una, sin embargo, clasificamos el perdón y desaferrarse del pasado como las primordiales en el campamento; además, saber tomar decisiones para estar mejor con nosotros mismo, para llevar una vida más sana, así como la importancia de tener una buena salud mental.

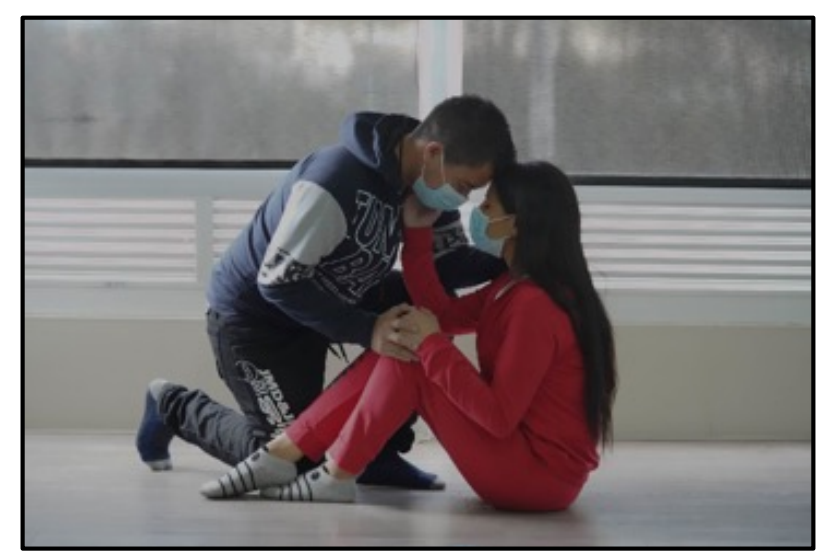

\section{Nombre de la escultura: "La pérdida"}

Creadores/as: John Alexis Alba Contreras, Angie Hernández, Diana Ramírez, Vanessa León

\section{Descripción}

Nuestra escultura representa la pérdida, en la que evidenciamos el amor, el apoyo, la tristeza; tal vez no necesitamos perder a alguien para vivir estas emociones, pero siempre necesitamos del otro, para que nos ayude a levantarnos y sea nuestro apoyo, ya sea la familia, la pareja o los amigos.

\section{Aprendizaje}

No importa la situación por la que estemos pasando: debemos tener en cuenta que no estamos solos, siempre habrá alguien dispuesto para ayudarnos a pasar por un duelo. Solo debemos alzar la cabeza, levantarnos, comunicar nuestro problema a la persona más cercana con la certeza de que me podrá escuchar y ayudar a superar cualquier crisis. No estamos solos, debemos afrontar nuestras situaciones o problemas con motiva- 
ción y esperanza al entender que el sentimiento no dura para siempre, es momentáneo si se sabe llevar de la mejor forma.

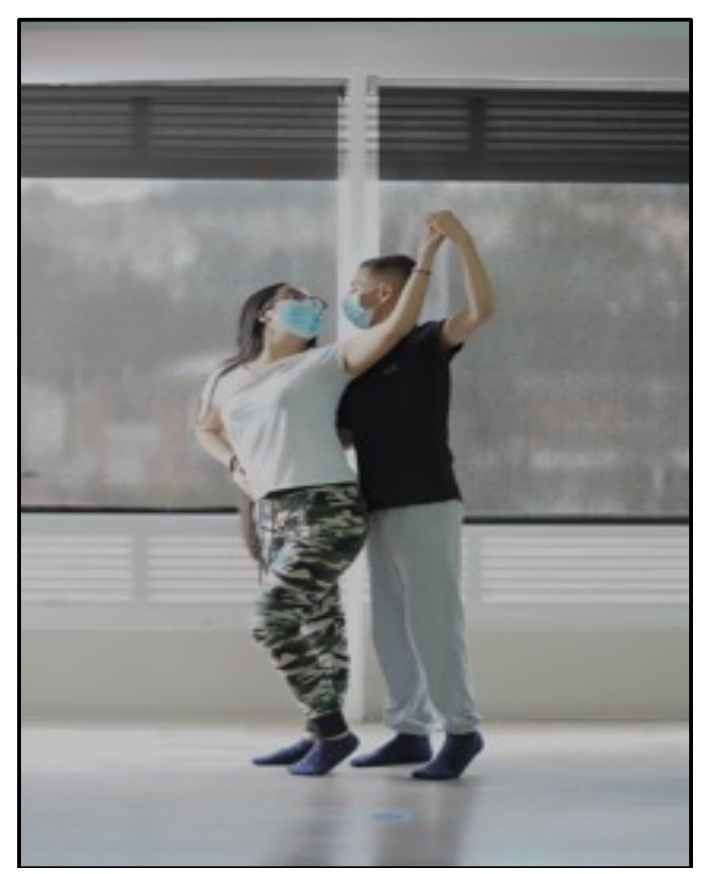

Nombre de la escultura: "La pasión de una danza" Creadores/as: Rafael Stiven Martínez Almeida, Nicol Vanessa Posada Sánchez, Ana Sofía Guarnizo Ochoa, Laura Daniela Moreno Cepeda

\section{Descripción}

La danza es la historia de dos individuos unidos por una razón: pasión y amor; la pasión de compartir el mismo gusto por la danza y el amor que surgió de ella. Los dos enamorados le muestran a su público la felicidad y compromiso tan grande que tienen al hacer lo que aman y con quien aman. La expresión corporal que escogimos como grupo fue llamada la pasión de una danza. Nuestro objetivo fue darle a conocer a nuestros compañeros, docentes e invitados las diferentes emociones y sentimientos que se pueden presentar y apreciar en una acción que a muchas personas les Ilama la atención, en este caso la danza; además, representarlo en una figura de expresión corporal. Las emociones y sentimientos que evidenciamos de nuestra escultura fueron alegría, tradición, técnica, estado físico, unión, sincronización, amor, pasión, protección, alegría y compromiso.

\section{Aprendizaje}

El ejercicio de tres días nos dio seguridad, confianza, amor por nosotros mismos y por los demás; nos enseñó el valor de trabajo en equipo, que las buenas acciones nos pueden ayudar en el presente, incluso mucho más en el futuro. Pudimos darnos cuenta de las cargas internas que cada persona lleva en su interior y lo difícil que es soltarlo o, incluso, compartirlo, pero de alguna forma algunos lograron tener esa fuerza y desahogarse, al soltar ese pasado oscuro que nos ata fuertemente. Los invitados nos dejaron la reflexión de nunca rendirse, que en la vida siempre habrá caminos complejos, pero debemos enfrentarlos y seguir adelante con el plan. Nunca rendirnos, incluso si llegamos a cumplir un reto, pues tenemos que seguir proponiéndonos aún más, porque de eso se trata la vida: de intentar una y otra vez.

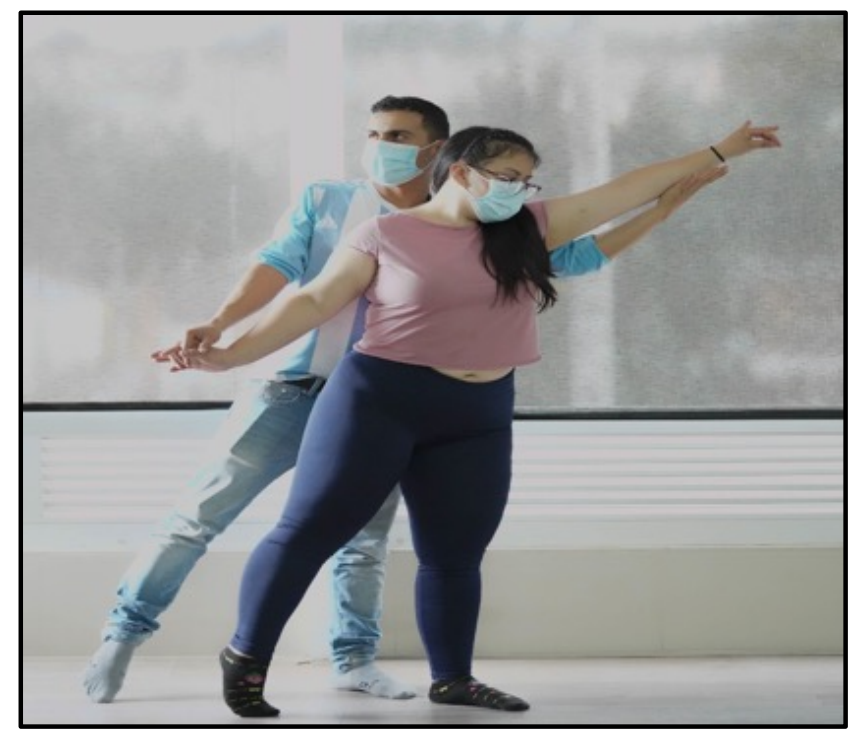

Nombre de la escultura: "El último baile"

Creadores/as: Andrés Ibagué, Brian Ramírez, Ángela Nova, Sebastián Rosero

\section{Descripción}

Una pareja se sitúa en un hermoso pub de los años ochenta. El hombre, cuyo propósito es ir a la guerra, desea estar una última noche con el amor de su vida, a quien diagnosticaron con leucemia. Así que se proponen ir a fumar y tomar unas copas de brandy, para abandonar un poco la realidad. La lluvia inundó aquel bar y el joven decidió bailar con su novia en la terraza del estableci- 
miento: puso una hermosa melodía y bailó junto a ella; rieron y disfrutaron de su último baile en una noche lluviosa junto a la luz de luna llena.

\section{Aprendizaje}

Se logró obtener un mejor manejo de las emociones, pues esta enseñanza era uno de los focos de interés y en lo que se quería centralizar a los estudiantes. Logramos replantear nuestros sueños y metas, al tener en cuenta lo que queremos cumplir a lo largo de nuestras carreras. Nos fue de mucha utilidad para dar el primer paso en depositar algo de confianza a esas personas que nos acompañarán a lo largo del pregrado, quienes serán los compañeros que tendremos en el camino hacia el éxito y formación como los mejores enfermeros. También fue de mucha ayuda para liberar todas esas emociones que teníamos atrapadas desde hacía mucho tiempo y que no sabíamos cómo expresar ante las demás personas.

\section{Discusión}

La Ley 1616 de 2013 (Congreso de Colombia, 2013) define la salud mental como "un estado dinámico que se expresa en la vida cotidiana a través del comportamiento y la interacción de manera tal que permite a los sujetos individuales y colectivos desplegar sus recursos emocionales, cognitivos y mentales (ejecutivos) para tramitar por la vida cotidiana, para trabajar, establecer relaciones significativas y para contribuir a la comunidad". En la experiencia del campamento, se revelan y fortalecen los recursos en mención, a través de vivencias con el cuerpo y el diálogo consigo mismo.

\section{El cuerpo y la relación intrapersonal}

El acercamiento al cuerpo, el lugar donde convergen las experiencias del ser, sucede por medio de los sentidos. El darse cuenta de manera consciente de lo que se ve, escucha y se siente dentro y fuera del propio cuerpo ha sido un medio frecuente hacia el autoconocimiento, tanto en disciplinas ancestrales como en las prácticas más modernas. Autores como Reinhard Flatischer (2005) y Diane Austin (2008) invitan a volcar la atención hacia la propia voz y la naturaleza rítmica musical del cuerpo, para llegar a estados de conciencia en los que se facilita el autoconocimiento, la expresividad de las emociones y la catarsis. La exposición receptiva a música ambiental, cuyos referentes modernos son la música incidental para cine, teatro o televisión, sobresale por su potencial sedativo o activador para ejercicios evocativos, que conducen a experiencias de reconocimiento emocional o autobiográfico. El cuerpo se vale de la música y lo sonoro como un puente que une los elementos del mundo invisible, como la imaginación, la memoria y la asociación, y nos traslada a la consciencia, como lo sugieren Pfeiffer y Zamani (2019).

El cuerpo ha sido sujeto de múltiples miradas teóricas a lo largo de la historia, entre ellas la perspectiva biomédica, propia de la biología, que concibe al cuerpo como un organismo en que operan funciones naturales; la perspectiva psicoanalítica, que lo concibe como un centro de energía, cuya circulación y expresión está facilitada o bloqueada por actitudes mentales; la perspectiva espiritual, que concibe el cuerpo como el lugar de habitación del alma, y la perspectiva existencial, que observa al cuerpo como existencia corporizada, el lugar desde y donde se organiza experiencialmente la existencia (Castro, 2011). Estas miradas invitan a reflexionar, en todo caso, en cómo el cuerpo constituye un correlato de la experiencia emocional y cognitiva, y, más allá, cómo el cuerpo es sistema en el que convergen y se dinamizan la experiencia del ser.

Estos abundantes desarrollos teóricos han abierto la puerta a la observación, el estudio y la práctica de la corporalidad en su relación con las demás dimensiones humanas, al comprenderla como una de las puertas de acceso a estas. Dan cuenta de ello la emergencia de sistemas de práctica como la Danza Movimiento Terapia, el Movimiento Vital Expresivo, el Teatro imagen y el Teatro del oprimido, entre otras, que hacen parte del acercamiento psicocorporal para el cuidado y la promoción del bienestar humano. Estas prácticas, que hacen parte de las técnicas performativas en la atención en salud, han probado su potencial en diversos escenarios de atención e, incluso, en Colombia, están integradas a las 
orientaciones metodológicas para la atención psicosocial a víctimas del conflicto armado (Ministerio de Salud y Protección Social, 2017).

Quien ejerce la enfermería se repliega sobre el cuerpo de cuidado y, a través de este, accede a la mente de las personas en momentos de gran conmoción; por lo tanto, la formación del talento humano en enfermería enfrenta el desafío que implica cuidar con el cuerpo y llegar al sujeto que se es, dentro de la experiencia de alto potencial humanizador que es cuidar. Si se ha tomado la opción de profesionalizar el cuidado, ¿cómo hacer de la experiencia de cuidar un camino hacia la realización personal? Comprender la vulnerabilidad y lo que entraña para la relación de cuidado, comprender el alivio de un oportuno contacto para el dolor y la fuerza de los silencios en diálogos inundados por la emoción pueden ser los recursos que un estudiante conquiste sin que nadie le señale, si ha tenido promoción del sujeto que está llamado a ser.

\section{La experiencia de dialogar consigo mismo}

El compromiso que reviste ser docente en salud mental ha permitido comprender que una didáctica efectiva para la formación de profesionales con capacidades para entablar relaciones terapéuticas requiere la combinación de la vivencia, conceptualización, práctica con otros/as y la gestión creativa. Dicho proceso de formación inicia en su etapa de fundamentación como proceso de desarrollo personal, en la experiencia de campamento sobre la relación intrapersonal. La realidad interna es siempre tan esquiva que, apoyada en el lenguaje, alumbra las verdades propias, para seguir los intereses o inquietudes y poder comprenderse al recorrer los propios terrenos una y otra vez, hasta encontrar esos tesoros de certezas que no están en ninguna otra parte, sino en la profundidad de la realidad interior. El diálogo consigo mismo es capaz de las más cálidas alianzas y expresiones de afecto, las palabras alcanzan tal intimidad, como solo es capaz la poesía.
En la experiencia del diálogo consigo mismo se resalta el concepto freudiano acerca del logro del niño, al ser capaz de expulsar el mundo exterior, al inaugurar los primeros pasos de la relación consigo mismo.

Por medio de ellas [las representaciones verbales] quedan convertidos los procesos mentales interiores en percepciones. Es como si hubiera de demostrar que todo conocimiento procede de la percepción externa dada una sobrecarga del pensamiento son realmente percibidos los pensamientos -como desde fuera- y tenidos así por verdaderos. (Freud, 1992)

De allí la importancia de ejercicios vivenciales, como el campamento señalado en el presente artículo, en el que el/la estudiante logra identificar su lenguaje interior y con ello fortalecer la relación consigo mismo/a como sujeto.

\section{Conclusiones}

El campamento nos da respuestas en la búsqueda por una didáctica específica para abordar la salud mental. La mente está inmersa en el cuidado y el estudiante necesita revisar su estado mental y fortalecerse. Al no ser un contenido de la cognición, la escuela ofrece espacios controlados y dirigidos para brindar una experiencia que promueva un encuentro consigo mismo en la observación de la realidad interior en procesos de autorreflexión que definitivamente le muestren el estado de la relación intrapersonal soporte para entablar procesos sostenibles de autocuidado.

En los cuidados de enfermería, accedemos a la mente a través del cuerpo. Desde primer semestre, necesitamos entendernos en nuestra corporalidad, reconocer en ella tanto la cultura como nuestra biografía, para de esta manera tener recursos mentales suficientes que nos permitan abordar los cuerpos de cuidado tremendamente presentes a causa de la enfermedad, desde la fuerza del sujeto. 
Lo anterior instala la construcción de aquello que cada quien requiere para entablar vínculos de cuidado requeridos para el ejercicio profesional de enfermería. Durante los semestres venideros, podrán profundizar en la relación intrapersonal que visibilizaron durante el presente campamento, con el consiguiente fortalecimiento como parte del hilo conductor de Cuidado y Calidad de Vida que promueve el programa de Enfermería de la Fundación Universitaria Juan N. Corpas, para así resaltar el cuidado de sí para cuidar a otros/as/es.

\section{Agradecimientos}

Estudiantes de primer semestre de Enfermería 2021-2022. Maritza Barroso Niño, enfermera, especialista, coordinadora de A. SALUDARTE y docente coordinadora del Campamento de Salud Mental 2021-2022. Juan David Guerrero Arbeláez, magíster en Música. Erika Alejandra Ramírez Gordillo, enfermera y magíster. Adriana González Vega, enfermera y magíster. Y Laura Olarte, bióloga y fotógrafa del campamento.

\section{Referencias Biblográficas}

1. Alonso, L. (2000). El papel del lenguaje interior en la regulación del comportamiento. Educere, 3(9), 61-68. https://www.redalyc.org/articulo.oa?id=35630911

2. Austin, D. S. (2008). The Theory and Practice of Vocal Psychotherapy: Songs of the Self. Jessica Kingsley Publishers.

3. Borgeaud-Garciandía, N. (2018). El trabajo de cuidado. Fundación Medifé. https://fundacionmedife.com.ar/sites/default/files/PDF/Edita/El\%20Trabajo\%20De\%20Cuidado-DIGITAL.pdf

4. Castro, A. M. D. (2011). Corporalidad en el contexto de la psicoterapia. 30.

5. Congreso de Colombia. (2013). Ley 1616 del 21 de enero de 2013. Por medio de la cual se expide la ley de salud mental y se dictan otras disposiciones. https://bit.ly/32oLZml

6. Cooper, M. (2003). «|-|» and «|-Me»: Transposing Buber's Interpersonal Attitudes to the Intrapersonal Plane. Journal of Constructivist Psychology, 16(2), 131-153.

https://doi.org/10.1080/10720530390117911

7. Flatischler, R. (2005). El poder olvidado del ritmo: TA KE TI NA. Mandala Ediciones.

Freud, S. (1984). El malestar en la cultura. Alianza Editorial.

8. Freud, S. (1992). El yo, el ello y otras obras (1923-1925) (vol. 19). Amorrortu editores.

https://www.bibliopsi.org/docs/freud/19\%20-\%20Tomo\%20XIX.pdf

9. Giraldo, L. F. G., y Zuluaga, C. G. (2013). El cuidado de sí y de los otros en Foucault, principio orientador para la construcción de una bioética del cuidado. Discusiones Filosóficas, 14(22), 15. http:// www.scielo.org.co/pdf/difil/v14n22/v14n22a12.pdf

10. Ministerio de Salud y Protección Social. (2017). Estrategia de Atención Psicosocial a Víctimas del Conflicto Armado. En el marco del programa de atención psicosocial y salud integral a víctimasPAPSIVI. Gobierno de Colombia. https://www.minsalud.gov.co/sites/rid/Lists/BibliotecaDigital/ RIDE/DE/PS/estrategia-atencion-psicosocial-victimas-conflicto-armado-papsivi.pdf 
11. Nespereira-Campuzano, T., y Vázquez-Campo, M. (2017). Inteligencia emocional y manejo del estrés en profesionales de Enfermería del Servicio de Urgencias hospitalarias. Enfermería Clínica, 27(3), 172-178. https://doi.org/10.1016/j.enfcli.2017.02.007

12. Pfeiffer, C., y Zamani, C. (2019). Explorando el cerebro musical. Musicoterapia, música y neurociencias | varios autores. Kier, editorial. http://www.elargonauta.com/libros/explorando-el-cerebro-musical-musicoterapia-musica-y-neurociencias/978-84-17581-63-3/

13. Piñeros Ricardo, A. M., Piñeros Ricardo, L. G., y Piñeros Ricardo, J. D. (2019). Corpas Humana, Corpas Social, Corpas Global. Proyecto Educativo Institucional. Fundación Universitaria Juan N. Corpas. https://www.juanncorpas.edu.co/fileadmin/Brenda_2/PEI_Corpas_Humana_Corpas_ Social_Corpas_Global_18112019.pdf

14. Rogers, C. R., y Wainberg, L. R. (2014). El proceso de convertirse en persona: mi técnica terapéutica. Paidós.

15. Román, M. A., Joya, F., y Becerra, A. C. (2015). Proyecto Educativo del Programa de Enfermería de la Fundación Universitaria Juan N. Corpas. Fundación Universitaria Juan N. Corpas. 